\title{
Constraints on inflation in the Einstein-Brans-Dicke frame
}

\author{
Yungui Gong * \\ Physics Department, University of Texas at Austin, Austin, Texas 78712
}

\begin{abstract}
The density perturbation during inflation seeds the large scale structure of the Universe. We consider both new inflation-type and chaotic inflation-type potentials in the framework of EinsteinBrans-Dicke gravity. The density perturbation gives strong constraints on the parameters in these potentials. For both potentials, the constraints are not much different from those obtained in the original inflationary models by using Einstein gravity.

98.80.Cq, 04.50. $+\mathrm{h}$
\end{abstract}

\section{INTRODUCTION}

The successful explanation of cosmological puzzles, such as the horizon, flatness, and monopole problems, is achieved by the inflationary scenarios. The inflationary scenarios also predict the spectrum of the density perturbation which seeds the formation of the large scale structure of the Universe. The basic assumption is that, at the early times, the Universe experienced an accelerated expansion, while the Hubble radius changed very slowly. Consequently the wavelength of a quantum fluctuation soon exceeds the Hubble radius. The amplitude of the fluctuation is frozen after the horizon crossing. After the end of inflation, the Hubble radius increases faster than the scale factor; so the fluctuations eventually reenter the Hubble radius during the radiation-dominated (RD) or matter-dominated (MD) eras. The original model, the so-called "old inflation" model [1], is based on the first-order phase transition. It failed because of the "graceful exit" problem. Soon after the "new" and "chaotic" inflationary models were proposed to solve this problem [2]. These models use a simple scalar field as the matter source. The scalar field (inflaton field) slow-rolls down the potential during the inflationary phase. All the above models are based on Einstein gravity. However, inflation may be driven by non-Einstein gravity. "Extended inflation" employs Jordan-Brans-Dicke (JBD) gravity [3]. The introduction of the Brans-Dicke (BD) field slows down the inflation and solves the graceful exit problem. But it was soon found that there was a "big bubble" problem for the original extended inflation [4]. The interaction between the BD field and the inflaton field can change the spectrum of the density perturbation. The spectra of density perturbations were analyzed in the extended new and chaotic inflationary models by several authors [5]. But the density perturbations given by those papers are not correct [6]. The correct density perturbation in BD inflation was given in [6] In this paper, I use the slow-roll approximation and work with BD gravity in the Einstein frame (let us call it Einstein-Brans-Dicke gravity). There are strong arguments identifying the Jordan frame as the physical one. The possibility of identifying the Einstein frame as the physical one was first raised by Cho [8]. Cho [8] and Damour and Nordtvedt [9] pointed out that only in the Einstein frame does the Pauli metric represent the massless spin-2 graviton and the scalar field represent the massless spin-0 field. In the Jordan frame the graviton is described by both the Pauli metric tensor and the BD scalar field. Cho also pointed out that in the compactification of Kaluza-Klein theory, the physical metric must be identified as the Pauli metric because of the wrong sign of the kinetic term of the scalar field in the Jordan frame. In string theory, the dilaton field appears naturally. The Einstein frame is greatly favored over the string frame although the string frame is chose for the pre-big-bang cosmology. For further discussions about the two frames, see [10] and references therein. Because the dilaton field evolves very slowly during the $\mathrm{RD}$ and $\mathrm{MD}$ eras, we then assume that the dilaton field at the end of inflation takes the same value as that at present. By this assumption, we can fix the value of the inflaton field at the beginning of inflation. We find that the results are different from those in [6]. Inflationary models based on general scalar-tensor gravity in the Einstein frame were also discussed in [11] $]^{3}$ and [12].

The BD Lagrangian in Einstein frame is

\footnotetext{
*Email address: ygong@physics.utexas.edu

${ }^{1}$ Susperregi pointed out to me that the density perturbations obtained in 6 and 77 are consistent although they were derived from two different approaches. I appreciate the comments made by him.

${ }^{2}$ Both the Pauli frame and the Einstein frame are used interchangeably in the literature.

${ }^{3}$ The author thanks David Wands for pointing out these references.
} 


$$
\mathcal{L}=\sqrt{-g}\left[-\frac{1}{2 \kappa^{2}} \Re-\frac{1}{2} g^{\mu \nu} \partial_{\mu} \sigma \partial_{\nu} \sigma\right]+\mathcal{L}_{m}\left(\psi, e^{-a \sigma} g_{\mu \nu}\right),
$$

where $\kappa^{2}=8 \pi G, a=\beta \kappa$, and $\beta$ is a constant. In this paper, I consider the simplest case, one simple scalar field as the matter source. The generalization to a more complicated multi-scalar-tensor gravity in Jordan frame was discussed in [9]. For the cosmological models in the context of general scalar-tensor theory in Jordan frame, see [13]. The matter Lagrangian is given by

$$
\mathcal{L}_{m}\left(\psi, e^{-a \sigma} g_{\mu \nu}\right)=-e^{-a \sigma} \sqrt{-g}\left[\frac{1}{2} g^{\mu \nu} \partial_{\mu} \psi \partial_{\nu} \psi+e^{-a \sigma} V(\psi)\right] .
$$

The energy-momentum tensor for this matter source is

$$
T_{(m) \mu \nu} \equiv \frac{2 \delta \mathcal{L}_{m}}{\sqrt{-g} \delta g^{\mu \nu}}=e^{-a \sigma} \partial_{\mu} \psi \partial_{\nu} \psi+g_{\mu \nu} \mathcal{L}_{m}=e^{-2 a \sigma}\left[(\rho+p) U_{\mu} U_{\nu}+p g_{\mu \nu}\right]
$$

where

$$
U_{\mu}=-\frac{\partial_{\mu} \psi}{\sqrt{-g^{\alpha \beta} \partial_{\alpha} \psi \partial_{\beta} \psi}}, \quad \dot{\psi}=\partial_{\mu} \psi U^{\mu}
$$

and $\rho=\frac{1}{2} e^{a \sigma} \dot{\psi}^{2}+V(\psi), p=\frac{1}{2} e^{a \sigma} \dot{\psi}^{2}-V(\psi)$. With the assumption that the scalar field $\psi$ (inflaton field) is spatially homogeneous, we can get the evolution equations of the Universe from the actions (1) and (2) based on the Robertson-Walker metric

$$
\begin{gathered}
H^{2}+\frac{k}{R^{2}}=\frac{\kappa^{2}}{3}\left(\frac{1}{2} \dot{\sigma}^{2}+e^{-2 a \sigma} \rho\right), \\
\ddot{\sigma}+3 H \dot{\sigma}=\frac{1}{2} a e^{-2 a \sigma}(\rho-3 p), \\
\ddot{\psi}+3 H \dot{\psi}=-e^{-a \sigma} V^{\prime}(\psi)+a \dot{\sigma} \dot{\psi},
\end{gathered}
$$

where $V^{\prime}(\psi)=d V(\psi) / d \psi$.

By the slow-roll approximations

$$
\frac{1}{2} e^{2 a \sigma} \dot{\sigma}^{2} \ll V(\psi), \quad \frac{1}{2} e^{a \sigma} \dot{\psi}^{2} \ll V(\psi), \quad \ddot{\sigma} \ll 3 H \dot{\sigma}, \quad \ddot{\psi} \ll 3 H \dot{\psi},
$$

Eqs. (4a)-4c for a flat universe become

$$
\begin{gathered}
H^{2} \approx \frac{\kappa^{2}}{3} e^{-2 a \sigma} V(\psi), \\
3 H \dot{\sigma} \approx 2 a e^{-2 a \sigma} V(\psi), \\
3 H \dot{\psi} \approx-e^{-a \sigma} V^{\prime}(\psi) .
\end{gathered}
$$

The consistency conditions for the above approximations are

$$
\beta^{2} \ll \frac{3}{2}, \quad \frac{e^{a \sigma} V^{\prime 2}(\psi)}{6 \kappa^{2} V^{2}(\psi)} \ll 1, \quad\left|\frac{e^{a \sigma} V^{\prime \prime}(\psi)}{3 \kappa^{2} V(\psi)}\right| \ll 1 .
$$

From Eqs. (6a)-60 $)$, we get

$$
\exp [a \sigma(\psi)]=2 \kappa^{2} \tilde{m}_{P l}^{2}\left(\frac{R(t)}{R(0)}\right)^{2 \beta^{2}}=2 \kappa^{2} \tilde{m}_{P l}^{2}\left[1-\frac{\beta^{2}}{\tilde{m}_{P l}^{2}}\left[g(\psi)-g\left(\psi_{i}\right)\right]\right],
$$




$$
H(\psi)=\sqrt{\frac{\kappa^{2}}{3}} e^{-a \sigma(\psi)} \sqrt{V(\psi)},
$$

where $g(\psi) \equiv \int d \psi V(\psi) / V^{\prime}(\psi)$ and $\tilde{m}_{P l}<m_{P l}$ is an arbitrary integration constant corresponding to the effective Planck mass at the beginning of inflation. Here I set the beginning of inflation to be $t=0$. Remember that the variation of the dilaton field $\sigma$ is very small during the MD era and the late times of the RD epoch. Although the dilaton field may have large changes during RD era for some initial values [10], we still assume that at the end of inflation the dilaton field becomes $\sigma\left(t_{e}\right) \approx \ln (16 \pi) / a$. Note that $e^{a \sigma}$ changes by a factor of $\left(T_{p} / T_{E Q}\right)^{\beta^{2}} \sim 10^{-5 \beta^{2}}$ only from the matter-radiation equality to the present. Therefore, from Eq. 8a , we get

$$
N_{t o t}=\ln \frac{R\left(t_{e}\right)}{R(0)}=\frac{1}{2 \beta^{2}} \ln \frac{m_{P l}^{2}}{\tilde{m}_{P l}^{2}} .
$$

It is easy to get $N_{t o t} \geq 65$. The conditions (7) tell us that inflation ends when

$$
m_{P l}^{2} V^{\prime 2}\left(\psi\left(t_{e}\right)\right) \approx 3 V^{2}\left(\psi\left(t_{e}\right)\right), \quad \text { or }\left|2 m_{P l}^{2} V^{\prime \prime}\left(\psi\left(t_{e}\right)\right)\right| \approx 3 V\left(\psi\left(t_{e}\right)\right) .
$$

The density fluctuation due to primordially adiabatic fluctuation is given by [6]

$$
\begin{aligned}
\frac{\delta \rho}{\rho} & =f \frac{H^{2}}{2 \pi}\left[\frac{e^{3 a \sigma / 2}}{16 \pi \dot{\psi}}+\frac{1-e^{a \sigma} /(16 \pi)}{\dot{\sigma}}\right]_{t_{k}} \\
& =f \frac{H}{2 \pi}\left[-\frac{e^{a \sigma / 2} \kappa^{2} V(\psi)}{16 \pi V^{\prime}(\psi)}+\frac{\kappa}{2 \beta}\left[1-e^{a \sigma} /(16 \pi)\right]\right]_{t_{k}},
\end{aligned}
$$

where $f$ equals $4 / 9$ if the fluctuations reenter the horizon during the $\mathrm{RD}$ era or $2 / 5$ if the fluctuations reenter the horizon during the MD era, and $t_{k}$ is the time at the horizon crossing during the inflationary epoch. For convenience, we use the number of $e$-foldings, $N_{k}$, before the end of inflation, instead of $t_{k}$. As usual, we take $N_{k} \approx 55-60$. In terms of $N_{k}$, we have

$$
\exp \left[a \sigma\left(t_{k}\right)\right] \approx 16 \pi \exp \left(-2 \beta^{2} N_{k}\right) .
$$

In order to go further, we need to specify the form of the potential.

\section{NEW INFLATION-TYPE POTENTIAL}

In this section, we take the Coleman-Weinberg potential [14],

$$
V(\psi)=\frac{B \eta^{4}}{2}+B \psi^{4}\left[\ln \left(\psi^{2} / \eta^{2}\right)-\frac{1}{2}\right]
$$

where $B \approx 10^{-3}, \eta \approx 2 \times 10^{15} \mathrm{GeV}$. The above potential can be well approximated by

$$
V(\psi) \approx \frac{B \eta^{4}}{2}-\frac{\lambda}{4} \psi^{4}=V_{0}-\frac{\lambda}{4} \psi^{4},
$$

where $V_{0}=B \eta^{4} / 2$ and $\lambda=\left|4 B \ln \left(\psi^{2} / \eta^{2}\right)\right|$ is approximately a constant. From the potential (13), we know that inflation will end when

$$
\begin{gathered}
m_{P l}^{2} V^{\prime 2}\left(\psi\left(t_{e}\right)\right) \approx 3 V^{2}\left(\psi\left(t_{e}\right)\right), \quad \text { if } \frac{1}{4} \lambda \psi^{4}\left(t_{e}\right)>\frac{3}{5} V_{0}, \\
\left|2 m_{P l}^{2} V^{\prime \prime}\left(\psi\left(t_{e}\right)\right)\right| \approx 3 V\left(\psi\left(t_{e}\right)\right), \quad \text { if } \frac{1}{4} \lambda \psi^{4}\left(t_{e}\right) \leq \frac{3}{5} V_{0} .
\end{gathered}
$$

The inflaton field at the end of inflation takes the value

$$
\psi_{f}^{2}=\psi^{2}\left(t_{e}\right) \approx-4 m_{P l}^{2}+\frac{2}{\lambda} \sqrt{\lambda V_{0}+4 \lambda^{2} m_{P l}^{4}} \approx \frac{V_{0}}{2 \lambda m_{P l}^{2}} .
$$


In the last step, we use the result $V_{0} \ll 4 \lambda m_{P l}^{4}$. Because $\lambda \psi_{f}^{4} / 4 \ll V_{0}$, it is well justified to approximate $V(\psi) \approx V_{0}$ during inflation. By using this approximation, we find the time evolutions of the scale factor, dilaton field, and inflaton field as

$$
\begin{gathered}
\exp [a \sigma(t)]=2 \kappa^{2} \tilde{m}_{P l}^{2}\left(1+2 \beta^{2} H_{v} t\right), \\
R(t)=R(0)\left(1+2 \beta^{2} H_{v} t\right)^{1 / 2 \beta^{2}}, \\
\frac{1}{\psi^{2}}=\frac{1}{\psi_{i}^{2}}-\frac{\lambda}{3 \kappa^{2} \tilde{m}_{P l}^{2} H_{v}} t=\frac{1}{\psi_{i}^{2}}-\frac{2 \lambda}{\sqrt{3 V_{0}} \kappa} t,
\end{gathered}
$$

where $H_{v}=\sqrt{V_{0}} / 2 \sqrt{3} \kappa \tilde{m}_{P l}^{2}$ is the Hubble parameter at the beginning of inflation. The function $g(\psi)$ is

$$
g(\psi)=\int d \psi \frac{V(\psi)}{V^{\prime}(\psi)} \approx-\int d \psi \frac{V_{0}}{\lambda \psi^{3}}=\frac{V_{0}}{2 \lambda} \psi^{-2} .
$$

Substituting Eqs. (14) and (15) back into Eq. (8a), we find that

$$
\begin{gathered}
\psi_{i}^{2}=\frac{\beta^{2} V_{0}}{2 \lambda\left[\left(1+\beta^{2}\right) m_{P l}^{2}-\tilde{m}_{P l}^{2}\right]} \approx \beta^{2} \psi_{f}, \\
\psi^{2}\left(t_{k}\right)=\frac{\beta^{2} V_{0}}{2 \lambda m_{P l}^{2}\left[1+\beta^{2}-\exp \left(-2 \beta^{2} N_{k}\right)\right]} .
\end{gathered}
$$

Therefore the density perturbation is

$$
\begin{gathered}
H\left(t_{k}\right)=\frac{1}{2 \sqrt{24 \pi}} e^{2 \beta^{2} N_{k}} \sqrt{\frac{V_{0}}{m_{P l}^{2}}}, \\
\frac{\delta \rho}{\rho}=\frac{1}{5 \sqrt{3} \pi \beta^{3}} e^{\beta^{2} N_{k}}\left[1+\beta^{2}-e^{-2 \beta^{2} N_{k}}\right]^{3 / 2} \sqrt{\lambda}+\frac{\sqrt{3}}{60 \pi \beta}\left(e^{2 \beta^{2} N_{k}}-1\right) \frac{\sqrt{V_{0}}}{m_{P l}^{2}} .
\end{gathered}
$$

Note that our formula is different from that in [6]. Take $N_{k}=60, \beta^{2}=0.001$ [15], then we get

$$
\frac{\delta \rho}{\rho}=47.6 \sqrt{\lambda}+0.04 \frac{\sqrt{V_{0}}}{m_{P l}^{2}} .
$$

The second term in the right hand side is much less than $10^{-5}$. Therefore, if we require $\delta \rho / \rho<10^{-5}$, we must have

$$
\lambda<4.4 \times 10^{-14} \text {. }
$$

Note that the minimum value of the coefficient before $\sqrt{\lambda}$ in Eq. $(17 \mathrm{~b})$ is about 38.4 when $\beta \approx 0.13$. If $\beta^{2} \geq 0.04$, the coefficient increases quickly and it requires smaller $\lambda$ to get the right density perturbation. In [16], the author did not get the constraint on $\lambda$ because they considered the fluctuation from the dilaton field only. However, it is clear form Eq. (17b) that the fluctuation from the inflaton field is larger than that from the dilaton field. In fact, our result is easily understood. Note that the fluctuation due to the inflaton field is

$$
\delta \psi(t)=\frac{e^{a \sigma(t) / 2} H(t)}{2 \pi} \leq \frac{1}{2 \pi} \frac{\sqrt{16 \pi}}{2 \sqrt{24 \pi}} \sqrt{\frac{V_{0}}{\tilde{m}_{P l}^{2}}}=\frac{1}{2 \pi} \sqrt{\frac{V_{0}}{6 \tilde{m}_{P l}^{2}}} .
$$

Here we use $\psi(t)>\psi_{i}$ and $\psi_{i}$ is given in Eq. 16a). To make our semiclassical discussions valid, we must require that the classical value of the inflaton field be larger than its quantum fluctuation. So we have

$$
\lambda<\frac{12 \pi^{2} \beta^{2} \tilde{m}_{P l}^{2}}{m_{P l}^{2}} .
$$

The smallness of the value of $\lambda$ cannot be saved. In [17, Matacz derived a different formula for the scalar quantum fluctuation $\delta \psi$ based on stochastic approach and gave a constraint $\lambda \sim 10^{-5}$. Perhaps that is one way to avoid the fine-tuning problem. 


\section{CHAOTIC TYPE POTENTIAL}

For chaotic inflation, the potential takes the power law type

$$
V(\psi)=\frac{\lambda_{n}}{n} \psi^{n}
$$

where $n$ is an even integer. In this case, the function $g(\psi)$ is

$$
g(\psi)=\int d \psi \frac{V(\psi)}{V^{\prime}(\psi)}=\int d \psi \frac{\psi}{n}=\frac{\psi^{2}}{2 n} .
$$

Because $n \geq 2$, the end of inflation will happen when

$$
\begin{gathered}
\left|2 m_{P l}^{2} V^{\prime \prime}\left(\psi\left(t_{e}\right)\right)\right| \approx 3 V\left(\psi\left(t_{e}\right)\right), \\
\psi_{f}^{2}=\frac{2 n(n-1)}{3} m_{P l}^{2} .
\end{gathered}
$$

Combining Eqs. (8a), 22), and (23b), we find

$$
\begin{gathered}
\psi_{i}^{2}=\left(\frac{2 n}{\beta^{2}}+\frac{2 n(n-1)}{3}\right) m_{P l}^{2}-\frac{2 n}{\beta^{2}} \tilde{m}_{P l}^{2} \approx\left(\frac{2 n}{\beta^{2}}+\frac{2 n(n-1)}{3}\right) m_{P l}^{2}, \\
\psi^{2}\left(t_{k}\right)=\frac{2 n}{\beta^{2}}\left(1+\frac{(n-1) \beta^{2}}{3}-\exp \left(-2 \beta^{2} N_{k}\right)\right) m_{P l}^{2} .
\end{gathered}
$$

In terms of $N_{k}$, we find

$$
\begin{aligned}
& H\left(t_{k}\right)=\frac{1}{2 \sqrt{24 \pi}} e^{2 \beta^{2} N_{k}}\left[\frac{2 n}{\beta^{2}}\left(1+\frac{(n-1) \beta^{2}}{3}-\exp \left(-2 \beta^{2} N_{k}\right)\right) m_{P l}^{2}\right]^{n / 4} \sqrt{\frac{\lambda_{n}}{n m_{P l}^{2}}}, \\
& \frac{\delta \rho}{\rho}=\frac{1}{5 \pi} \sqrt{\frac{\lambda_{n}}{n}} m_{P l}^{n / 2-2}\left[h\left(N_{k}\right)\right]^{n / 4}\left|-\frac{1}{2 n \sqrt{6}} e^{\beta^{2} N_{k}} \sqrt{h\left(N_{k}\right)}+\frac{1}{4 \sqrt{3} \beta}\left(e^{2 \beta^{2} N_{k}}-1\right)\right|
\end{aligned}
$$

where

$$
h\left(N_{k}\right)=\frac{2 n}{\beta^{2}}\left(1+\frac{(n-1) \beta^{2}}{3}-\exp \left(-2 \beta^{2} N_{k}\right)\right)
$$

For $n=2$, we have

$$
\frac{\delta \rho}{\rho}=1.7 \frac{\sqrt{\lambda_{2}}}{m_{P l}}
$$

So the bounds on the anisotropy of the microwave background give

$$
\lambda_{2}<4 \times 10^{-11} m_{P l}^{2} .
$$

For $n=4$, we have

$$
\frac{\delta \rho}{\rho}=30.7 \sqrt{\lambda_{4}}
$$

In order to get the small density perturbation, we require

$$
\lambda_{4}<1 \times 10^{-13} \text {. }
$$


At last, let us look at the exponential potential $V(\psi)=V_{0} \exp \left(-\psi / \psi_{0}\right)$ [18]. In this case, both $V^{\prime}(\psi) / V(\psi)$ and $V^{\prime \prime}(\psi) / V(\psi)$ are constants. Then the slow-roll condition cannot determine when the inflation will end under the assumption that $\exp (a \sigma)=16 \pi$ at the end of inflation. Therefore, this kind of potential is not workable in our concern. In this model, we must consider the interaction between the inflaton field and other fields to let the Universe exit from the inflationary epoch. In [7], the authors considered the exponential potential in extended inflation. They used the consistency conditions (7) to give the value of the BD field at the end of inflation. Since we know the evolution of the BD field during the RD and MD eras, it may be a problem to match the value of the BD field from the end of inflation to the present. We would like to say a few more words about the difference between our results and those in [6]. As a result of the assumption that the dilaton field takes the same value at the end of inflation and the present, we can fix the value of the inflaton field at both the beginning and the end of inflation. If we take the approximation $\exp \left(-2 \beta^{2} N_{k}\right) \approx 1-2 \beta^{2} N_{k}$, then our results $(17 \mathrm{~b})$ and $(25 \mathrm{~b})$ are similar to those in [6]. For the choices of $\beta^{2}$ and $N_{k}$ in this paper, we can take this approximation. That is why our numerical values do not differ much

from those in $[6]$. Instead of thinking the physical frame to be the Jordan frame, we work in the Einstein frame.

\section{ACKNOWLEDGMENTS}

The author would like to thank Professor Yuval Ne'eman for his helpful comments.

[1] A. Guth, Phys. Rev. D 23, 347 (1981).

[2] A. Linde, Phys. Lett. 108B, 389 (1982); A. Albrecht and P. Steinhardt, Phys. Rev. Lett. 48, 1220 (1982); A. Linde, Phys. Lett. 129B, 177 (1983).

[3] D. La and P. J. Steinhardt, Phys. Rev. Lett. 62, 376 (1989); P. J. Steinhardt and F. S. Accetta, ibid. 64, 2740 (1990); D. La, Phys. Rev. D 44, 1680 (1991).

[4] E. Weinberg, Phys. Rev. D 40, 3950 (1989); D. La., P. J. Steinhardt and E. W. Bertschinger, Phys. Lett. 231B, 231 (1989); A. Liddle and D. Wands, Mon. Not. R. Astron. Soc. 253, 637 (1991); Phys. Rev. D 46, 3655 (1992).

[5] J. McDonald, Phys. Rev. D 44, 2314, (1991); S. Mollerach and S. Matarrese, ibid. 45, 1961 (1992); N. Deruelle, C. Gundlach and D. Langlois, ibid. 46, 5337 (1992); J. García-Bellido, A. Linde and D. Linde, ibid. 50, 730 (1994); J. García-Bellido, Nucl. Phys. B423, 221 (1994).

[6] A. Starobinsky and J. Yokoyama, in Proceedings of the 4th Workshop on General Relativity and Gravity, Kyoto, Japan, 1994, edited by K. Maeda, T. Nakamura and K. Nakao, gr-qc/9502002, p. 381; T. Chiba, N. Sugiyama and J. Yokoyama, Nucl. Phys. B530, 304 (1998).

[7] M. Susperregi, Phys. Rev. D 55, 560 (1997); M. Susperregi and A. Mazumdar, ibid. 58, 083512 (1998).

[8] Y.M. Cho, Phys. Rev. Lett. 68, 3133 (1992).

[9] T. Damour and K. Nordtvedt, Phys. Rev. D 48, 3436 (1993); D. Santiago, D. Kalligas and R. Wagoner, ibid. 58, 124005 (1998).

[10] D. Wands, Class. Quantum Grav. 11, 269 (1994); Y. Gong and Y. Z. Zhang, Int. J. Mod. Phys. D 4, 333 (1995); R. Dicke, Gen. Relativ. Gravit. 30, 435 (1998); Y. Gong, gr-qc/9809015.

[11] J. García-Bellido and D. Wands, Phys. Rev. D 52, 6739 (1995); 53, 5437 (1996).

[12] R. Holman, E. W. Kolb, S. L. Vadas and Y. Wang, Phys. Rev. D 43, 3833 (1991); T. Damour, G. W. Gibbons and C. Gundlach, Phy. Rev. Lett. 64, 123 (1990); T. Damour and C. Gundlach, Phys. Rev. D 43, 3873 (1991).

[13] J. Barrow, Phys. Rev. D 47, 5329 (1993); 50, 3746 (1994).

[14] S. Coleman and E. Weinberg, Phys. Rev. D 7, 1888 (1973).

[15] Y. Gong and Y. Z. Zhang, Europhys. Lett. 31, 7 (1995).

[16] A. L. Berkin, K. Maeda and J. Yokoyama, Phys. Rev. Lett. 65, 141 (1990); A. L. Berkin and K. Maeda, Phys. Rev. D 44, 1691 (1991).

[17] A. Matacz, Phys. Rev. D 56, 1836 (1997).

[18] D. Schwarz and J. Martin, astro-ph/9805313. 